\title{
Ultraviolet Studies of Cepheids
}

\author{
Erika Böhm-Vitense ${ }^{1,2}$ \\ ${ }^{1}$ University of Washington, Seattle, Washington, ${ }^{2}$ Guest observer with the \\ International Ultraviolet Explorer (IUE) Satellite
}

\begin{abstract}
We discuss whether with new evolutionary tracks we still have a problem fitting the Cepheids and their evolved companions on the appropriate evolutionary tracks. We find that with the Bertelli et al. tracks with convective overshoot by one pressure scale hight the problem is essentially removed though somewhat more mixing would give still better fits.

Using results of recent nonlinear hydrodynamic calculations by Morgan we find that we also have no problem matching the observed pulsation periods of the Cepheids with those expected from their new evolutionary masses, provided that Cepheids with periods less than 9 days are overtone pulsators.

We investigate possible mass loss of Cepheids from UV studies of the companion spectrum of S Mus and from the ultraviolet spectra of the long period Cepheid $l$ Carinae. For $\mathrm{S}$ Mus with a period of 9.6 days we derive an upper limit for the mass loss of $\mathrm{M}<10^{-9} \mathrm{M}_{\odot}$, if a standard velocity law is assumed for the wind. For $l$ Carinae with a period of 35.5 days we find a probable mass loss of $\mathrm{M} \sim 10^{-5 \pm 2} \mathrm{M}_{\odot}$.
\end{abstract}

\section{Background}

The Cepheid mass problem has been with us for a long time. Around 1980 standard evolution theory predicted for Cepheids with pulsation periods around 10 days an evolutionary mass around $7 \mathrm{M}_{\odot}$ for solar abundances, while pulsation theory and especially the bump Cepheids required masses between 4 and $5 \mathrm{M}_{\odot}$, see Cox 1980 .

Ultraviolet observations with the IUE satellite revealed a number of Cepheid companions with temperatures $\leq 10,000 \mathrm{~K}$, see Böhm-Vitense and Proffitt (1985). Several of them had radii larger than expected for main sequence stars. They appeared to be giants. Since evolution time scales for giants are very short they should then have masses which are only a few percent less than their Cepheid primaries. They should fit essentially on the same evolutionary track as the Cepheids. They were, however, observed to be much fainter and indicated masses of the order of the pulsational masses. In Table 1 we give the basic data for the Cepheids and their evolved companions as given by Böhm-Vitense and Proffitt 1985 in their Table 6C. We did apply a correction of $\Delta \log \mathrm{L}=0.2$ to all luminosities in order to match the Sandage and Tammann (1969) distance scale.

In Figure 1 we show the positions of the Cepheids and their companions in the HR diagram together with the newer evolutionary tracks of Becker (1981) with no 
convective overshoot. It is obvious that we had a problem, (except for AX Circini which seems to fit well).

Table 1

Data for Cepheids and companions used here

\begin{tabular}{llllrrr} 
Star & $\log \mathrm{P}$ & $\begin{array}{l}\log \mathrm{T}_{\text {eff }} \\
(\mathrm{Ceph})\end{array}$ & $\begin{array}{l}\log \mathrm{L} \\
(\mathrm{Ceph})\end{array}$ & $\begin{array}{r}\log \mathrm{T}_{\text {eff }} \\
(\operatorname{comp})\end{array}$ & $\begin{array}{r}\log \mathrm{L} \\
(\operatorname{comp})\end{array}$ & $\Delta \log \mathrm{L}$ \\
\hline AX Cir & 0.722 & 3.76 & $3.48($ ov) & 4.17 & 3.54 & -0.06 \\
AW Per & 0.810 & 3.73 & $3.40(\mathrm{ov})$ & 4.08 & 2.87 & 0.55 \\
SV Per & 1.046 & 3.72 & 3.50 & 4.08 & 3.11 & 0.39 \\
SY Nor & 1.102 & 3.69 & 3.36 & 4.04 & 2.75 & 0.61 \\
RW Cam & 1.215 & 3.67 & 3.45 & 4.08 & 2.99 & 0.46 \\
KN Cen & 1.532 & $?$ & 4.27 & 4.40 & 3.40 & 0.87
\end{tabular}

The numbers in brackets give the deviations for the two sets of data.
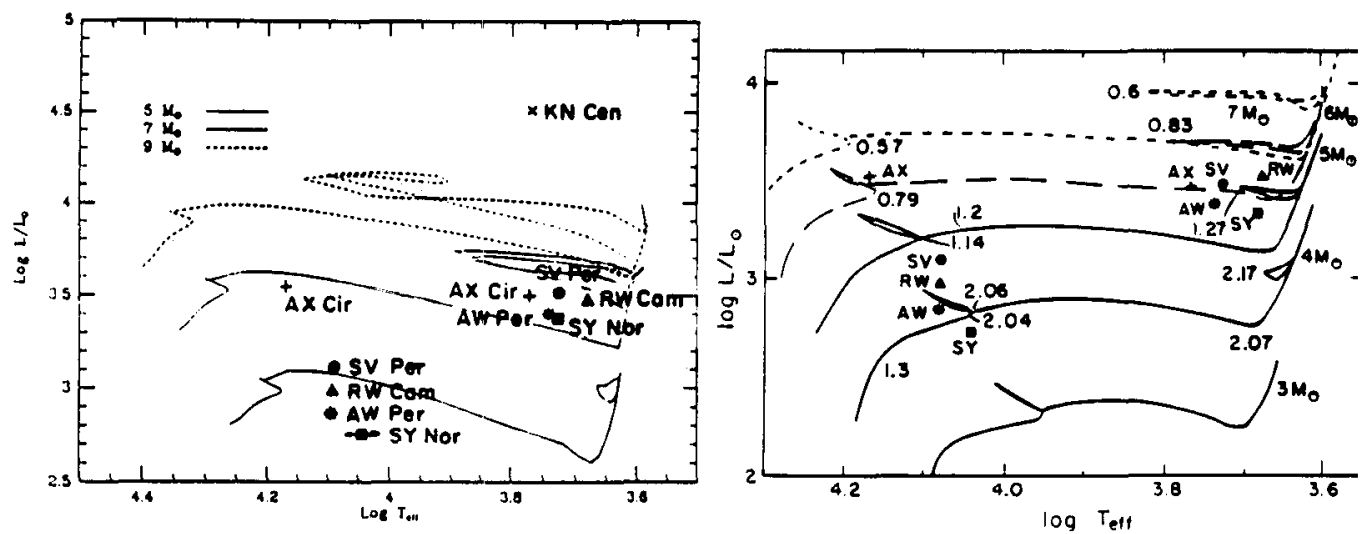

Figure 1 (left) Evolutionary tracks without convective overshoot are shown for 5, 7, and $9 \mathrm{M}_{\odot}$ stars according to Becker (1981). Also shown are the positions of the Cepheids and their "giant" companions according to Böhm-Vitense and Proffitt (1985) with L increased by 0.2 dex. Cepheids and evolved companions do not fit on one isochrone. AX Cir is the exception.

Figure 2 (right) Evolutionary tracks with convective overshoot by one pressure scale height are shown for $3,4,5,6$, and $7 \mathrm{M}_{\odot}$ stars according to Bertelli et al. (1986). Also shown are the positions of the Cepheids and their evolved companions. For these tracks the companions fit on the evolved part of the main sequence where evolution times are longer. The masses of the companions now come out to be about $10 \%$ smaller than those of the Cepheids (except for AX Cir). A minute increase in the overshoot length would place them on one isochrone. 


\section{New evolutionary tracks}

Smaller Cepheid masses are one way to eliminate the mass problem for Cepheids. This requires that for a given mass the blue loops on which the Cepheids are believed to be found must have higher luminosities. Becker and Cox (1982) pointed out that increased mixing in the interiors of the main sequence progenitors would increase the luminosities of the blue loops. It would also explain the high nitrogen to carbon abundance ratios found for supergiants (Luck and Lambert 1981; Mena-Werth 1992). Bertelli, Bressan, Chiosi and Angerer (1986) followed up on that possibility and calculated a number of evolutionary tracks with overshoot above the convective core.

Maeder and Meynet (1988) calculated evolutionary tracks for overshoot by 0.3 pressure scale heights and some mass loss. In Figure 2 we show the Bertelli et al. evolutionary tracks for convective overshoot by one pressure scale height. We also show the positions of the Cepheid binaries with evolved companions. The evolved companions now fit on the evolved part of the main sequence where the evolution is still slower than on the giant branch. Their masses appear to be only 10 to $15 \%$ less than the Cepheid masses, which are about $4.8 \pm 0.3 \mathrm{M}_{\odot}$. Considering the error limits the fit is quite good though a somewhat higher luminosity for the blue loops would give an even better match. (Tracks with the new OPAL opacities (Iglesias and Rogers 1991, give loops with somewhat lower not higher luminosities, see Stothers and Chin 1992.) The loops need to extend further to the blue than shown by the Bertelli et al. tracks in order to reach to the Cepheid positions. Alongi et al. (1991) showed, however, that this can be achieved by also considering overshoot at the bottom of the outer convection zone by about 0.5 to 0.7 pressure scale heights.

AX Circini is an exception. It must be a first crossing star if the Bertelli et al. tracks are correct, or it must follow the Becker track, which means it must have had a progenitor which had no additional mixing. Of course we do not know whether the mixing is due to convective overshoot or to a different mechanism.

N. Evans (this volume) reaches a different conclusion. She concludes that no evolutionary tracks match all the Cepheids with evolved companions. She emphasizes the discrepant results for AX Cir and SU Cyg (see below) and also BP Cir if the Bertelli et al. tracks are used. Three first crossing stars out of 10 are too many according to evolution times unless there is a special selection mechanism. All three stars have periods less than 4 days and they have rather high temperatures. Such high temperatures may not be reached by the blue loops for low mass Cepheids. We can then observe such short period Cepheids only for first crossing stars. If we see indeed first crossing stars, then they should still have solar nitrogen to carbon abundance ratios. It would be quite interesting to check.

Another possibility is, of course, that the excess mixing does not occur for all stars. In that case we probably have to look for another mixing mechanism than convective 
overshoot, which should be similar for all stars of a given mass and composition.

\section{Pulsation properties}

The next question is whether such Cepheids have the theoretically expected pulsation periods. Pulsation periods for solar abundance Cepheids with different combinations of $\mathrm{L}$ and $\mathrm{T}_{\text {eff }}$ were calculated recently by Buchler, Moskalik and Kovacs (1990) and also by Morgan in Seattle (1991). We use here the results by Morgan. In Figure 3 we show the relations between period and $\mathrm{T}_{\text {eff }}$ for different luminosities. Also shown are the positions of the Cepheids according to their luminosities and periods. Since the exact $T_{\text {eff }}$ of these Cepheids are hard to determine because of the color contamination due to the bright companions we have plotted them at the best fitting values for $\mathrm{T}_{\text {eff }}$. (In Figures 1 to 3 we also used these $\mathrm{T}_{\text {eff }}$ ). For lower luminosities higher temperatures can be obtained. $\Delta \log \mathrm{L} \sim-0.1$ would raise the temperature by about $350 \mathrm{~K}$.

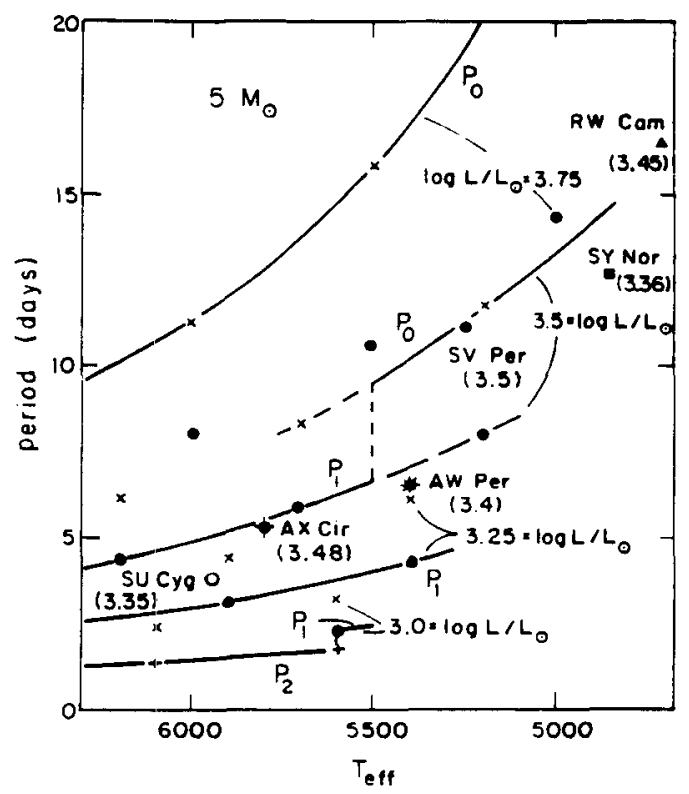

Figure 3 For given luminosities of the Cepheids the relation between periods and effective temperatures are shown according to non-linear hydrodynamic calculations by Morgan (1991). The fundamental periods $P_{o}$ are indicated by $x$, the first overtone periods $P_{1}$ by dots and the second overtone $\mathrm{P}_{2}$ by crosses. The prevailing modes are connected by solid lines. The dashed lines show the connections and the extensions for the non-prevailing modes. The Cepheid symbols are placed according to their luminosities and their periods. The $\log \mathrm{L}$ of the Cepheids are given in brackets below the symbols. AW Per and AX Cir must be overtone pulsators to fit on this diagram with reasonable temperatures.

Morgan could not find any fundamental mode pulsators with periods less than 9.5 
days. This agrees with our suggestion that for periods around 9 days a transition from fundamental mode pulsation to first overtone pulsation occurs for galactic Cepheids (Böhm-Vitense 1990). In Figure 3 the curves are drawn through the points that represent the prevailing mode of pulsation in nonlinear hydrodynamic modeling. We can fit AX Cir and AW Per on this diagram only if they are first overtone pulsators. As such they fit very well. It then seems that the discrepancy between evolutionary and pulsational masses has disappeared. If we adopt the Bertelli et al. tracks the Cepheid masses agree with the pulsation and bump masses.

One point of importance: If galactic Cepheids with periods less than 9 days are overtone pulsators we have to use different period luminosity relations for the two groups of stars as shown in Figure 4 taken from Morgan's thesis. For a given period and $\mathrm{T}_{\text {eff }}$ there is a shift in luminosity by about 0.2 dex. The overtone pulsators being the brighter ones as compared to the fundamental pulsators. For the fundamental mode pulsators the gradient of the period luminosity relation increases. This is important if we try to observe bright, long period Cepheids in other galaxies.

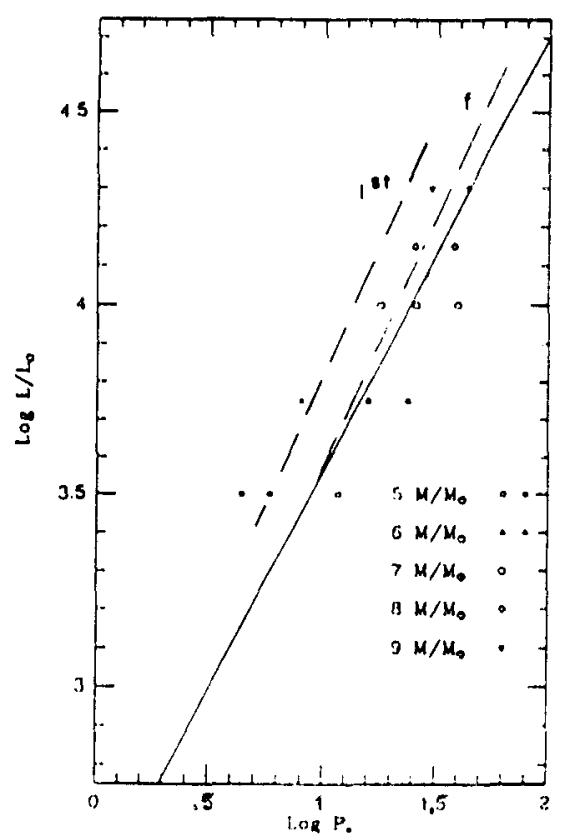

Figure 4 The relation between luminosities and periods is shown for stars of different masses and effective temperatures according to Morgan (1991). The open symbols refer to fundamental mode pulsators, the filled symbols to first overtone pulsators. The solid line gives the empirical relation according to Stothers and Carson. For a given period and $T_{\text {eff }}$ the overtone pulsators have a luminosity larger by 0.2 dex as compared to the fundamental mode pulsators. For each mode of pulsation alone we will derive a steeper period luminosity relation than for the combined set of fundamental and first overtone pulsators as suggested schematically by the dashed lines added by us. 
For AX Cir and AW Per, which we believe are overtone pulsators, we increased the luminosities by 0.2 dex to take this into account. This does not influence the luminosity difference between Cepheid and companion.

\section{Dynamical mass determinations}

Nancy Evans and I have tried very hard to determine dynamical masses for Cepheid binaries with main sequence companions. In Table 2 I have collected the results of our measurements. Within the error limits the results agree with the masses we just derived using the Bertelli et al. tracks except for SU Cyg. With its period of 3.8 days it has a larger mass than S Mus with a period of 9.6 days. We saw here that SU Cyg is expected to be an overtone pulsator. It fits on the evolutionary tracks only if it is at a first crossing. Both, the AX Cir and SU Cyg binaries, fit well on the blue loops of the Becker tracks. Possible explanations were discussed above.

Table 2

\begin{tabular}{lrrrll} 
Star & Period & Mode & $\begin{array}{r}\text { Mass } \\
\text { Bertelli }\end{array}$ & $\begin{array}{r}\text { Mass } \\
\text { dynamic }\end{array}$ & Reference \\
\hline S Mus & 9.6 & fund. & $5.4 \pm 0.2$ & $5.3 \pm 0.7$ & Böhm-Vitense et al. 1990 \\
SU Cyg & 3.8 & 1st & $4.8(6.0)$ & $>5.8 \pm 0.4$ & Evans and Bolton 1988 \\
V636 Sco & 6.0 & 1 st & $4.7 \pm 0.2$ & $4.8 \pm 0.5 ?$ & Böhm-Vitense 1986
\end{tabular}

\section{Mass loss of S Mus}

Willson and Bowen (1986) suggested that pulsating stars may lose mass very efficiently. If so, they might have started out with larger masses. We have attempted to determine the mass loss for the Cepheid binary S Mus. If the Cepheid is losing mass the wind passes by the companion and causes absorption lines in the companion spectrum, see Figure 5. We (Rodrigues and Böhm-Vitense 1992) tried to study these absorption lines in the companion spectrum for $\mathrm{S}$ Mus. The available IUE spectra were taken at an orbital phase when the stars were at maximum separation, $\left(\Phi=90^{\circ}\right)$. Liliya Rodrigues calculated the line profiles which would be expected for these wind absorption lines in the companion spectrum assuming a standard velocity law $\mathrm{v}(\mathrm{r})$ and $\Phi=90^{\circ}$. The largest absorption is due to particles near terminal velocities because of the long column density for this velocity. Near the center of the line the observed line profiles are confused by interstellar absorption and possibly Cepheid absorption lines or perhaps lines from S Mus B. We therefore studied mainly the broad, shortward shifted absorption component expected to be seen for such a passing cool Cepheid wind. The detailed structure of the profile is wiped out by the large turbulence expected to be present in the wind. 


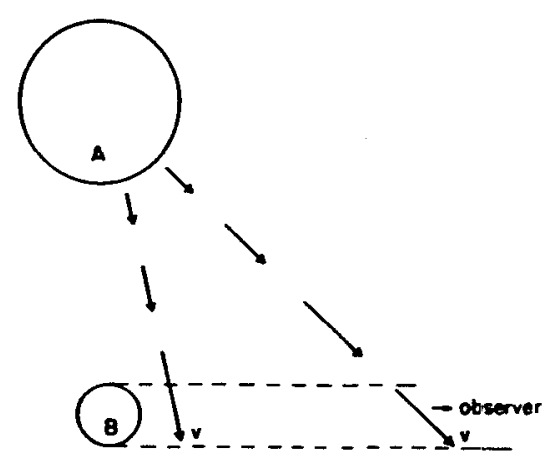

Figure 5 The geometry is shown for the S Mus system at the orbital phase of our observations. The Cepheid wind passes by the $\mathrm{B}$ star companion and causes absorption lines in its spectrum. The largest radial velocities are seen for the largest distance from the B star.
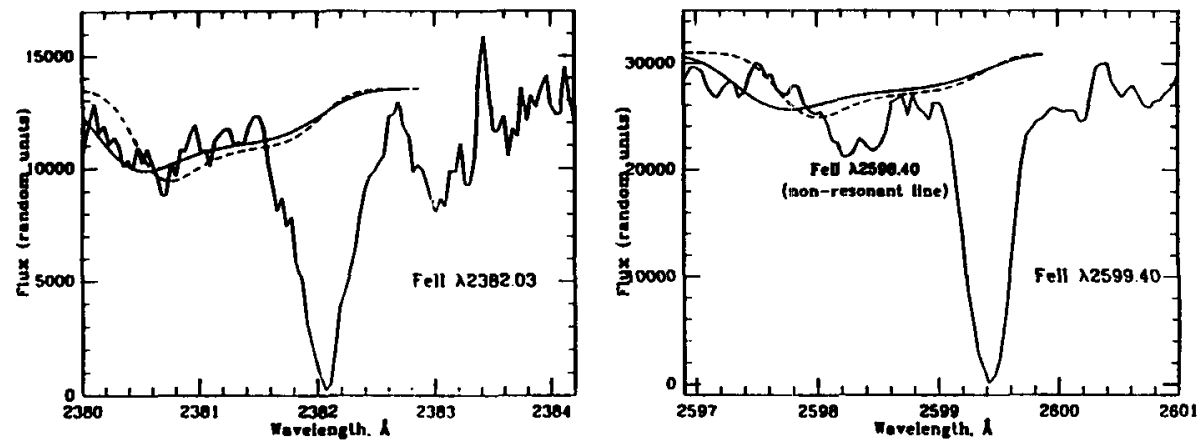

Figure 6 Observed and best matching theoretical line profiles for some Fe II lines in the B star companion spectrum are shown. The theoretical profiles correspond to a wind with a terminal velocity of $175 \mathrm{~km} / \mathrm{s}$ and a turbulent velocity of $37 \mathrm{~km} / \mathrm{s}$. The depth of the shallow satellite lines determines the density in the wind.

For several Fe II lines such broad shallow blue shifted satellite lines were observed with the relative strengths as might be expected according to the oscillator strengths. In Figure 6 we show the comparison of observed Fe II line profiles with the best matching theoretical ones for a terminal wind velocity of $175 \mathrm{~km} / \mathrm{s}$ and a turbulent velocity of $37 \mathrm{~km} / \mathrm{s}$. For these values and assuming that all $\mathrm{Fe}$ is present as Fe II we find a mass loss of about $10^{-10} \mathrm{M}_{\odot}$ per year. In order to check whether Fe could be present also as Fe III we looked at the Al III lines and found no sign of a blue satellite line. The Al III lines are expected to be weaker because of different abundances and 
different oscillator strengths. We therefore can conclude only that the amount of $\mathrm{Fe}$ III has to be less than about a factor 6 more than Fe II. This gives an upper limit for the mass loss of $6 \times 10^{-10} \mathrm{M}_{\odot}$ per year. This upper limit also holds if the broad shallow line should be intrinsic to S Mus B.

\section{Mass loss of $l$ Carinae}

It may be interesting to check whether we see evidence of stronger mass loss for Cepheids with longer periods. For $l$ Carinae with a period of 35.5 days a large number of high resolution long wavelength ( $2000 \AA-3000 \AA)$ and low resolution (1200 $\AA-2000 \AA$ ) spectra are available. The Mg II lines are always in emission. They are highly variable during the pulsation period. The profiles and their variations can be understood if at the wavelengths for maximum emission, i.e. at about $\lambda_{o} \pm 75 \mathrm{~km} / \mathrm{s}$, the emission occurs in an optically thick layer and shortly after maximum light in an optically thin layer. In addition there is some essentially constant absorption outside of this emitting layer. For this absorption we find at the wavelengths of maximum flux, i.e. at $\pm 75 \mathrm{~km} / \mathrm{s}$, an optical depth of about $\tau_{\lambda}=0.6$ independent of phase. If the turbulent velocity is about $30 \pm 5 \mathrm{~km} / \mathrm{s}$. and if $\mathrm{Mg}$ is all in the form of $\mathrm{Mg}$ II we derive a column density of $\mathrm{n}_{\mathrm{H}}=10^{21 \pm 2} \mathrm{~cm}^{-2}$. In order to estimate how much mass is in this "shell" we have to know its volume, which means its radius.

We believe that we see spatially extended emission in the OI lines, which leads to an estimate for the radius of the surrounding shell or cloud of about $1000 \mathrm{AU}$, i.e. the same order of magnitude as found by Mc Alary and Welch (1986) for some Cepheids with infrared excess.

Assuming a homogeneous sphere with the given column density and an outflow velocity of $\mathrm{v}_{\mathrm{r}} \sim 35 \mathrm{~km} / \mathrm{s}$ we estimate a mass loss of $\mathrm{M} \sim 10^{-5 \pm 2} \mathrm{M}_{\odot}$ per year. This is what would be expected according to Reimers' approximate relation for luminous stars.

It thus seems possible that mass loss may be of importance for longer period Cepheids. The uncertainties in our estimates (mainly of the turbulent velocity in the shell) are too large to reach a definite conclusion. Our studies seem to show a similar trend as seen by Deasy and Butler (1986) who found that short period Cepheids ( $\mathrm{P}$ $<15$ days) do not show excess infrared emission, while a few Cepheids with longer periods do. $l$ Carinae does, however, not show excess infrared emission.

\section{References:}

Alongi, M., Bertelli, G., Bressan, A.,and Chiosi, C. 1991, Astr. Astrophys., 244, 95.

Becker, S. and Cox, A. N. 1982, Ap.J., 260, 707.

Becker, S. A. 1981, Ap. J. Suppl., 45, 475.

Bertelli, G., Bressan, A., Chiosi, C., and Angerer, K. 1986, Astr. Astrophys. Suppl., 66, 191.

Böhm-Vitense, E. and Proffitt, C. 1985, Ap. J., 296, 175.

Böhm-Vitense, E., Clark, M., Cottrell, P. L. and Wallerstein, G. 1990, A.J., 99, 353.

Böhm-Vitense, E., 1986, Ap. J., 303, 262. 
Böhm-Vitense, E. 1990, Ap. J., 324, L27.

Böhm-Vitense, E. and Love, S. 1992, submitted to Ap. J.

Buchler, J. R., Moskalic, P. and Kovacs, G. 1990, Ap. J., 351, 617.

Chiosi, C., Wood, P. R., Bertelli, G., Bressan, A., and Mateo, M. 1992, Ap. J., 385, 205 .

Chiosi, C. and Wood, P. R. 1990, referenced by Chiosi, C. 1990, in ASP Conference Publ. 11,158 .

Cox, A.N . 1980, Ann. Rev. Astr. Astrophys., 18, 15.

Deasy, H. P. and Butler, C. J. 1986, Nature, 320, 726.

Evans, N. R. and Bolton, C. T. 1990, Ap. J., 356, 630.

Iglesias, C. A. and Rogers, F. G. 1991, Ap. J., 371, 408.

Maeder, A. and Meynet, G. 1988, Astr. Astrophys., 76, 411.

Mc Alary, C. W. and Welch, D. L. 1986, A. J., 91, 1209.

Morgan, S. 1991, Ph.D. Thesis, University of Washington, Seattle, WA.

Rodrigues, L. and Böhm-Vitense, E. 1992, Ap.J., Dec. 20.

Sandage, A. and Tammann, G. A. 1969, Ap. J., 157, 683.

Stothers, R. B. and Chin, C. W. 1991, Ap. J., 381, L67.

Willson, L. A. and Bowen, G. H. 1986, Ir. Astron. J., 17, 2.

\section{Acknowledgements}

This research was supported by NASA grant NSG 5398, which is gratefully acknowledged. I am very much indebted to the staff of the IUE Observatory. Without their continuous help for obtaining and reducing the data this study would not have been possible.

\section{Discussion}

Comment by Nancy Evans: I am going to talk about several of the same stars as Erika, and although I disagree with many of the details of the analysis and interpretation, I think a lot of her study was driven by the difficulty of matching systems with a Cepheid and an evolved companion with current isochrones. I find I have a similar difficulty with these systems.

Comment by S. R. Sreenivasan: 1. Rotational mixing due to differential rotation is an alternative for producing core growth in stars to a uniform overshoot postulated for all stars. This way the extend of core growth is proportional to the vigor of differential rotation and does not occur for all stars as you require. 2. Mass loss can occur in these Cepheids because of the large outer (or subsurface) convection zones.* It might also occur in the main sequence phase for stars whose mass is larger than $5 \mathrm{M}_{\odot}$ on the ZAMS if they have significant rotation. In that case surface rotational speed also undergoes evolutionary changes.

*due to both magnetic as well as non-magnetic (e.g. acoustic energy flux) causes. 